\title{
Swarming behaviour in elite race bunch cycling: a case study
}

\section{Introduction}

There has been a recent emergence of complex systems theory within sports performance analysis literature. Of particular note is that of dynamic patterning, principally based upon the conceptual framework provided by previous authors such as Haken (1983) and, subsequently, the model posited by Haken et al. (1985). This relates to the somewhat controversial supposition (see Lebed, 2006) that spatio-temporal patterns may characterise a sports contest as a self-organising dynamic system, within which the system's elements (players/athletes) demonstrate periods of co-operative interaction (McGarry et al. 2002). The seminal work of McGarry and co-authors (McGarry, et al. 1999; McGarry et al., 2002; McGarry and Perl, 2004; McGarry, 2006) has realised the potential of the sports contest as a dynamical system, commonly illustrated by way of racket sports such as squash and, more recently, tennis (Palut and Zanone, 2005). As such, the classical 'In-Phase' and 'Anti-Phase' patterning (stable attractor states) have been reported in racket sport dyads, reflecting the spatio-temporal harmony of players' coupled movements about the court surface (McGarry et al., 1999). The presence of a stable attractor state is considered to exist within dyadic sporting contests and describes the conformance of two competing opponents towards a co-ordinated movement pattern, matched in relative space and corresponding time frames. Such patterns are referred to as 'self organising' since these emerge independent of external instruction. Patterns are thought to maintain stability until perturbed by some destabilising event, otherwise known as a 'perturbation' (Hughes et al., 1998; Hughes et al., 2001; McGarry et al., 2006). Consequently, dyadic sports are widely considered to be more than the sum of two competing separate entities, rather, the product of a reciprocal interactive system that is drawn towards reaching stable attractor states, at least for transient periods during match play.

Within the general context of complex systems theory, reports from previous research have been instrumental in realising the value of understanding specific in-game behaviours which can be associated with perturbations (Hughes et al., 1998; Hughes et al., 2001). A perturbation 
is an event which serves to disrupt the reciprocal rhythm and subsequent stability of a system (i.e. two interacting players or teams). The attainment of such information is desirable since it may greatly reduce the amount of data derived from an analysed performance (Hughes, 2004; Lames and McGarry, 2007; McGarry, 2009) by identifying the potential causes of instability leading to critical incidents (Hughes et al., 1998; Hughes et al., 2001). For example, Hughes et al. (1998) reported upon the effect of perturbations, identified from incidents such as intercepting tackles or penetrating passes leading to a goal scoring opportunity in soccer (referred to as critical incidents). Any incident that changes the state of a systems' stability represents a perturbation, which may or may not result in a critical outcome.

More recently, some authors have supplemented research pertaining to dynamical systems in sport, theorising models of increased complexity, characterised by highly intricate interactions at the team level. For example, Reed and Hughes (2006) suggested an alternative approach to dynamical systems enquiry, describing the notion of 'momentum' and the associated 'rate of change' in the momentum of successful behaviours as a method of demonstrating collective stability within a rugby match. Such research has laid a foundation for mapping the continuum of stability-instability within team sport, moving away from traditional 'reductionist' accounts of dynamical systems (Grehaigne et al., 2005). Reed and Hughes were able to demonstrate the influence of perturbing incidents on changes in game momentum, highlighting the value of novel approaches to dynamic systems research.

The current paper sought to liken the dynamics of a bunch cycling race, namely the Points Race, to the emergent, self-organising theory of Swarming (Gazzi and Passino, 2004; Chu et $a l ., 2006)$. Bunch race cycling is a generic term used to describe all cycling events in which a collective grouping of individual riders occurs, often in a seemingly random fashion. The Points Race is an example of a bunch cycling event, comprising of a $40 \mathrm{~km}$ racing distance (Olympic and World Championship), taking place on a $250 \mathrm{~m}$ bevelled track. A sprint for points every 10 laps offers the event an additional higher intensity element. An intermittent convergence of individual riders into the aforementioned bunch formation, followed by a subsequent divergence, is also a noteworthy characteristic of the Points Race. Points are awarded for finishing within the top four riders (1-5 points) upon every $10^{\text {th }}$ lap (sprint lap) or, alternatively, lapping the field (20 points). Likewise, 20 points are deducted for being lapped by the leading bunch of the race. 
Within the Points Race, secondary group configurations or breakaways are frequently adopted, in which riders break-away from the main bunch often in a grouped linear formation, or as individuals, seemingly perturbing the stability (normal profile) of the group. These bunching tactics are collectively preferred by competitors as it enables a significant reduction of the individual drag coefficient $\left(C_{\mathrm{d}}\right)$ and, therefore, the work required to sustain a position in the race (Atkinson et al., 2003; Edwards, 2007). The comparison of the Points Race to swarming theory appears credible since the central concepts that characterise a swarm favourably correspond to the spatio-temporal dynamics of a bunch cycling race.

In most biological swarms, for example flocks of birds or swarms of bees, a mobile central locus point is assumed to exist at the epicentre of interplay between a "long-term attraction" and a "short term repel" of the group towards and away from the group centre, respectively (Gazzi and Passino, 2004, pg. 1). Such 'macro' effects reside upon an environmental transfer of 'micro' information between agents of the swarm (Parish et al., 2002), for example, visual or audio cues. Thus, the constant attraction-repulsion of the collective group represents the normal, stable state of the swarm. It is theorised that this behaviour reflects both the perceived protection of the group centre and the coupled contrasting need to detach from the group in order to fulfil alternative singular roles (Parrish et al., 2002). Whilst the comparison of race bunch cycling to swarming theory may appear obscure, the same concept of serial aggregation may indeed apply to the riders of the points race, since the longer term attraction and short term repel of the group is explained by the paradoxical position a rider experiences. That is, the long term attraction to the field, which is required to achieve the energy reductions associated with group formations and, conversely, the short term repulsion from the bunch, which is demonstrated as a consequence of attempting to gain the most credible race position. From this perspective, much like the central premise of swarming behaviour, a Points Race cyclist is unable to achieve the ultimate global outcome without drawing upon the micro interactions occurring between swarming agents.

There remains no empirical evidence addressing the bunching of riders in competitive situations as a dynamical system and, subsequently, little quantitative data to inform coaches of the most effective tactical approaches to deploy within this form of racing. In order to describe sports competitions involving mass athlete interaction as a dynamical system, it 
appears feasible to look beyond the narrowing concepts currently provided within sports of a dyadic nature. Therefore, to develop a greater understanding of the internal logic that underpins points race configurations, behavioural analysis software was specifically adapted to, firstly, identify a race profile of a basic swarm mentality within the Points Race and secondly, to identify system stability and the possible perturbation of stability, in relation to successful and unsuccessful breakaways.

\section{Method}

\subsection{Design}

A three stage design was adopted in which an initial intra-observer validation and subsequent identification of successful and unsuccessful breakaways was undertaken (see statistical analyses section for details). Both the second and third stages were undertaken concurrently, with the second stage consisting of an analysis of the two previously identified performance breakaway indicators using computerised analysis software (Dartfish TeamPro, 4.0.9.0, Switzerland). The third concurrent stage involved a dynamical systems investigation of the field of riders, utilising the swarming theory of serial aggregation. This was performed using identical analysis software (see following sections for procedures).

\subsection{Sample}

A case study was performed on 24 international track cyclists competing in a competitive points race World Championship event (2009). The race was performed on a standard sized wooden track (Indoor Velodrome, Poland) with a 250 metre internal radius, consisting of 160 laps of the track. Twenty Four cyclists started the race, however, this number often fluctuated between laps (i.e. 23 or 22) due to rider injury or unforeseen technical problems. Race duration reached 47 minutes and 39 seconds (based on the last rider). Ethical approval for this study was acquired from the University of Chester Ethics Committee for the Faculty of Applied Health Sciences.

\subsection{Identification of Performance Indicators (Independent Variable)}

Initial meetings between the lead researcher (over 300 hours experience with analysis systems) and an experienced track cycling analyst (5 years experience as a track cycling 
analyst) were held in which the consideration of breakaways as a potential cause of perturbation was raised. The breakaway was considered as a suitable performance indicator given the clear advantages a cyclist may gain when co-existing within a group formation and the current dearth of performance analysis research in track cycling.

Operational definitions for breakaways and the respective sub-types therein were created in the form of a definition dictionary (James et al., 2005; Worsfold and MacBeth, 2009). This initially consisted of defining the 'breakaway' and was further refined into definitions for both successful and unsuccessful breakaways. For example;

Breakaway - "The movement of an individual or a group away from the pre-formed race bunch, via the increase in velocity of the breaking group or a decrease in velocity of the original bunch. The initialisation of this movement is recognisable by a discernable time duration between the two formed bunches, judged to be outside of the average spacing of riders. This larger duration between riders must be maintained for a minimum of 5 seconds to be considered as a breakaway, this is otherwise considered as a minor fluctuation. The magnitude of distance between group bunches, velocity/accelerations of group bunches and time duration of a break may vary considerably. The membership of this break may also vary, with riders moving to and from the main bunch or breakaway bunches."

Both successful and unsuccessful breakaway occurrences were identified in an identical manner, deeming a breakaway as successful if the opportunity of scoring points came from the active involvement of a rider therein. A breakaway was otherwise deemed unsuccessful. Subsequently, breakaways were considered as the independent variable, comprising of two levels; unsuccessful and successful. The end of a breakaway was defined as the reforming of the front 'breakaway' group to the collective bunch, thus demonstrating no de-stabilisation of the race norm.

\subsection{Identification of Performance Indicators (Dependent Variables)}

The numerical group centre (i.e. middle rider) of the riders was chosen as the epicentre of distribution around which long term attraction and short term repel phases would occur. Long term repulsion was defined as the development of distance between riders (as a whole), resulting in a larger average group spacing. Attraction was defined as the opposite effect, in 
which the continual reduction in the average distance between riders was demonstrated. This was deemed appropriate since, during the Points Race, the protective properties of the bunch are well established and recognised by competitors. As a result, two separate groups are formed, the rear and the front group, each containing twelve riders (dependent upon the complement of riders at that half lap). The average duration of each group of riders from the numerical group centre upon each half lap was then used to describe the state of interaction with the whole system at that given stage, reflecting the normal movement to and from the group centre (i.e. attract and repel phase).

Three measures were created to profile the state of the swarm. These are abbreviated and herein referred to as;

1)

Absolute Difference (AbDiff) - Combined value of the mean duration of riders in the front group from the numerical group centre and the mean duration of riders in the rear group from the numerical group centre. Consequently, the magnitude of field distribution can be averaged (mean).

2)

Rate of Change (ROC) - The rate at which the group changes from one state of absolute difference to another within a given phase (see overleaf).

\section{$\underline{\operatorname{AbDiff}(x)+\operatorname{AbDiff}(y)}$}

Phase $D U R$

Where $\operatorname{AbDiff}(x)=$ initial absolute difference; $\operatorname{AbDiff}(y)=$ final absolute difference; $D U R=$ the number of half laps completed during that phase.

3) Phase Duration (DUR) - Number of half laps completed in a phase

\subsection{Analysis Procedure}

A three camera synchronised recording of the race was utilised in order to detect breakaways throughout the field of cyclists at any time in the race. The race was analysed post event using the tagging function on the computerised software. A template was designed in which, firstly, the 'breakaway' performance indicators (successful/unsuccessful) were recorded (stage 2 of 
design). A continuous event button was used to denote the event of a breakaway, with the feature of a 'successful' or 'unsuccessful' outcome assigned as an additional value. If an event was judged to be unclear or missed by the analyst, the event was replayed and re-analysed until a breakaway was deemed successful or unsuccessful.

In order to perform the second stage of analysis (stage 3 of design), the swarming of the group was analysed by using the continuous event option on the pre-designed template. The group was numerically divided in to two equal halves (12 riders each side), denoting the front and rear of the swarm. Under even numbers, the point of equal time duration between the twelfth and thirteenth rider was chosen to reflect group centre. Upon each half lap, denoted by the intersection of the lead rider's wheel with the half way lines (back and home straight), a time split between each member of the respective groups (front or rear) was recorded. The split of a rider was, again, identified by the intersection of the leading wheel with the half way lines. Computations of the time duration of each rider from the numerical group centre were subsequently carried out on Microsoft Excel (2003). Further computations were used to produce the average time duration between each rider at each half lap interval. The three dependent measures (absolute difference, rate of change and phase duration) were then sourced from this information.

\subsection{Statistical Analyses}

Intra-observer reliability was measured using Cohen's Kappa coefficient $(K)$ to provide a level of agreement with regards to the outcome of the breakaway (i.e. successful or unsuccessful). O’Donoghue (2007) suggests an interpretation of; $0.4-0.6=$ moderate strength, $0.6-0.8=$ good agreement and $>0.8$ = very good agreement. Results from $K$ suggested a 'very good agreement' of intra-observer reliability for break outcome $(K=0.891)$. This is important since the concurrent second and third stages of testing resided upon attaining a strong level of agreement.

Data comparisons were performed on each of the three dependent variables (AbDiff, ROC and DUR) using separate one way analyses of variance (ANOVA) with repeated measures. 
The independent variable consisted of three levels (Successful, Unsuccessful and 'Norm' (race mean)). The assumptions of normality (Shapiro Wilk; $>0.05$ ) and sphericity (Mauchly's; $>0.05)$ were verified for all data sets. The mean of each of the three dependent measures (AbDiff, DUR and ROC) for the race was used as a reference for the normal race profile (norm). Significant differences above or below the race mean or 'norm' is suggestive of a swarm status that is outside of the norm and, thus, perceived to be in a larger state of instability (in response to perturbation). Post-hoc Tukey HSD tests were applied in order to identify significant differences. Statistical significance was set at $P<0.05$ throughout.

\section{Results}

Figure 1 demonstrates the aggregated movement of the field of riders upon each half lap. An attract and repel phasing is evident, with each plot of both rear and front group timing demonstrating a consistent movement towards and away from the group centre. Throughout, such aggregative effects appear in an almost uniform fashion. On the basis of the race profile shown in figure 1, AbDiff, ROC and DUR were calculated.

Figure 1. Swarming trace of the attraction and repel of the front and rear group about the numerical group centre as a function of race time. $N B$ : Black dashes represent the average duration of the twelve riders of that group (front) from the numerical group centre upon each half lap. Open circles represent the rear group average (these are not negative values but have been assigned as such for presentation purposes). Black triangles represent the position of the lead rider upon each sprint lap. 
Results from the $3 \mathrm{x}$ one-way ANOVA's demonstrated no significant effect for any of the comparisons of $\operatorname{ROC}(F(2,228)=3.976, P>0.05)$ and $\operatorname{AbDiff}(F(2,228)=3.289, P>0.05)$ between successful, unsuccessful and norm conditions. However, there was a significant effect found for DUR $(F(2,228)=18.4, P<0.05)$. Tukey HSD post-hoc analysis revealed differences between the race norm (2.1 \pm 1.3 half laps $)$ and the successful ( $3.3 \pm 1.2$ half laps) and unsuccessful $(3.1 \pm 1.5$ half laps $)$ DUR of phases. This suggests a larger phase duration of either attracting or repelling compared to normal race values. All other values were nonsignificant $(P>0.05)$. See figures 2,3 and 4 for graphical representations of the mean comparison results and associated standard deviations.

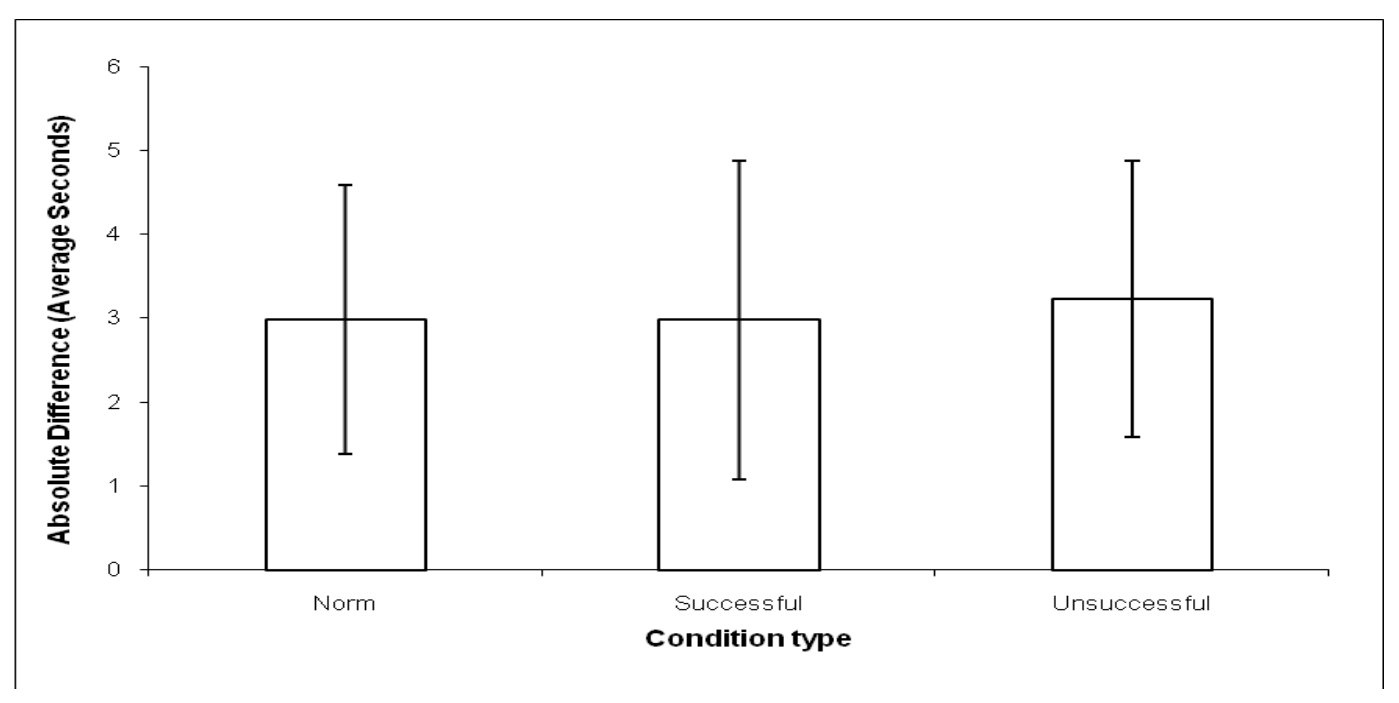

Figure 2. Comparison of mean Absolute Difference of successful and unsuccessful break opportunities against the race norm 


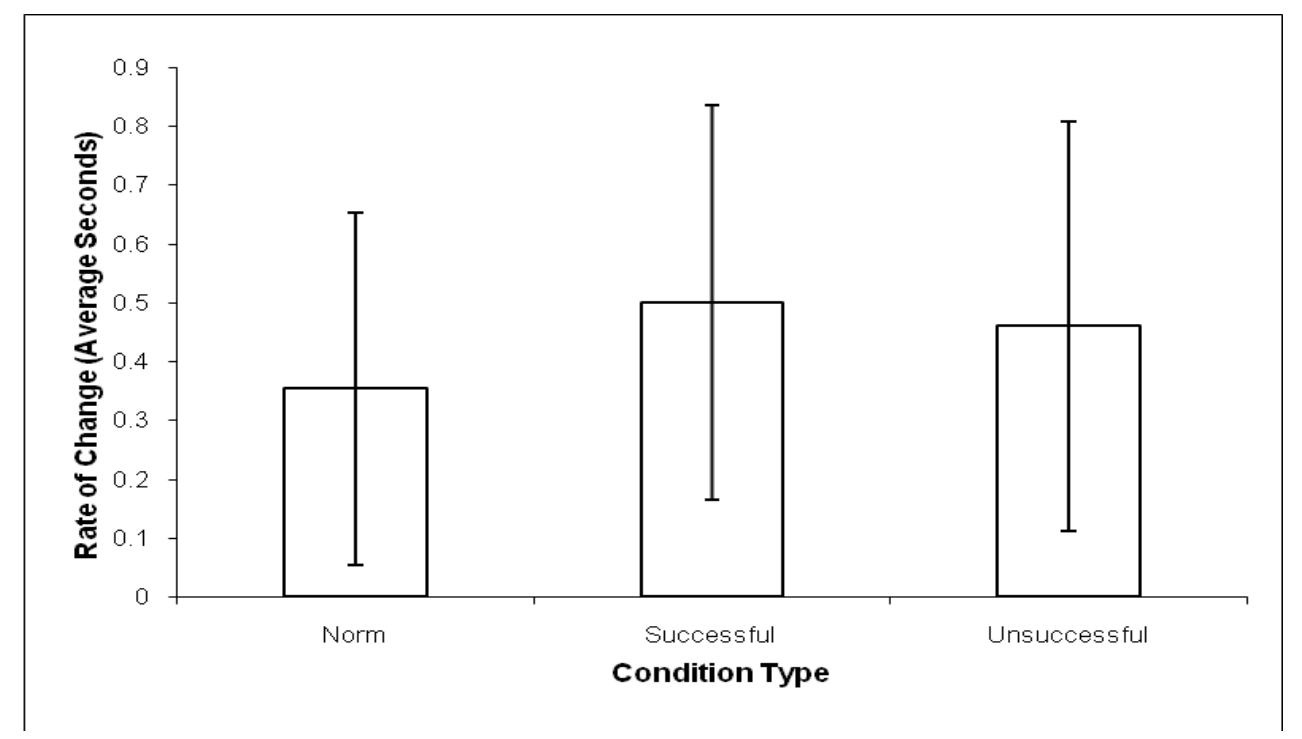

Figure 3. Comparison of mean Rate of Change of successful and unsuccessful break opportunities against the race norm

Figure 4. Comparison of mean Phase Duration of successful and unsuccessful break opportunities against the race norm. $N B: *=$ statistically significant from norm at $P<0.05$

\section{Discussion}


The initial aim of the current study was to create a system using generic performance analysis software that could demonstrate a race profile of the dynamics of the Points Race in reference to the swarm mentality. Although addressing this question remains somewhat descriptive, that figure 1 shows the field distribution as a function of race time provides evidence of the constant attract and repel phasing of the field, demonstrating a 'normal' rhythm to the race with potential areas of disturbance. The results were used to establish the stability of the system, producing figures 2, 3 and 4 and the associated 'norms' for each dependent variable. It is likely that there are alternative ways to interpret the swarming mentality demonstrated in figure 1, nonetheless, the results offer a basis with which to perform the current study and are a clearly attractive prospect for future research.

One way ANOVA comparisons between the race norm and both successful and unsuccessful breakaways demonstrated no differences $(P>0.05)$ with regard to whole field distribution of the swarm, represented by AbDiff (see figure 3). The same was true for ROC, where no significant difference $(P>0.05)$ from the race norm was reported (see figure 4$)$. Consequently, for any breakaway period (on average), neither the whole field distribution (Abdiff) or the rate at which the field moves from phase to phase (ROC) was statistically different from the race norm (mean for that race). This suggests no clear perturbation of the system in terms of these two properties during a breakaway. However, the DUR for both successful and unsuccessful breakaways demonstrated a significantly larger value $(P<0.05)$ than the race norm, with results showing phases of attract and repel to be over 3 half laps on average (see figure 5) compared to the race norm of $2.1 \pm 1.3$ half laps. This suggests that system instability in both successful and unsuccessful breaks is caused in terms of the length of attract and repel phases of the swarm, meaning that the field of riders tends to move together or apart for a longer duration (DUR) when a breakaway is occurring.

The findings from DUR comparisons provide interesting results since it appears that a breakaway causes some disturbance outside the 'norm', suggesting that there may be some perturbation to the system when a breakaway occurs. This further suggests that when a possible perturbation occurs, as a result of a breakaway, the aggregated field diverges for a longer period of time, outside the norm, without any associated change in the rate at which field distribution expands and without any initial difference in absolute field distribution. This 
is representative of a large informational transfer (i.e. visual) between the agents of the system (riders), producing a non-linear effect which serves to lock the swarm into an on-going repel phase. Conversely, under 'normal' race conditions the phase is likely to maintain an attractor state which, in this case, is an almost constant and statistically briefer attract and repel rhythm.

Figure 1 also demonstrates the position of the eventual race winner upon each sprint lap of the entire race. A notable trend for the repulsion of the group to increase concurrent with the race winners position at the front of the race would appear to show some influence of individual decisions upon the swarm mentality. Therefore, when such potentially influential riders make the decision to break-away from the main bunch, the chaotic effect is demonstrated through prolonged repulsive phases. Such knowledge is potentially valuable to a Points Race competitor where the desire may be to plan an effective tactical approach, basing the racing strategy upon favoured riders who it seems may exert an influence on the overall field mentality. The continuing search for perturbation in race bunch cycling may also be supplemented by information such as the decision making of individuals within the race. Future investigations should attempt to elucidate the relationship between the analysis of perturbation and the swarming mentality in contexts such as race bunch cycling or alternative sporting events that involve similar group patterning.

Whilst it is clear that the decisions of individual riders may influence the field of riders, a limitation of the current paper is the failure to establish a more refined cause of perturbation. Although a breakaway appears to cause a perturbation, and this exact point is determinable (see reliability section), a more detailed analysis of the breakaway may provide further information regarding the exact actions required to change the stability of the race. For example, further analysis of the membership or position of breakaways provide potential avenues for future investigation. The attainment of such results would bring this type of systemic enquiry closer to that of previous research (McGarry et al., 1999; McGarry, 2006) where the relationship between perturbation and key tactical behaviours can be more accurately realised. 
The application of the 'swarm mentality' within race bunch cycling remains within its infancy and, consequently, making accurate conclusions and inferences regarding racing tactics remains a distant objective. For example, it may be crude to identify instability as a significant different value of AbDiff, ROC or DUR from the race mean. Indeed, the lack of difference in the chosen dependent variables may merely reflect this potential limitation. An alternative method may be to identify the status of instability (or otherwise) as a scaled continuum, with stable and unstable conditions at opposing extremes. In turn, a profiling method similar in concept to Reed and Hughes (2006), where percentile ranks were used to denote movement outside of a pre-conceived range, may provide greater flexibility in showing stability and the associated variability of a normal race profile. In the current case, a significant difference away from the norm may be too harsh of a measure to detect instability accurately.

\section{Conclusion}

In conclusion, the current study confirmed the theory that a mass aggregation effect occurs with the Points Race, theoretically as a consequence of an informational transfer between individual riders, causing a non-linear 'macro' effect which can be described using computerised analysis software. Although linking tactical implementations to the current analysis remain a distant aspiration at present, the preliminary findings suggest that the breakaway causes disruptions to race stability and that the decisions of individual riders may influence such an occurrence. The correct application of future research may provide points race cyclists' and coaches with useful information regarding the exact actions required to initiate a successful breakaway, ultimately serving to cause perturbations and the resultant occurrence of a 'critical incident' within a given race (Hughes et al., 1998). It should be remembered that the current paper is prototypal, designed to explore the possibilities of dynamic system enquiry in sport at the level of mass aggregation, namely the swarming effect. As such, the current paper provides a clear methodological basis with which to perform future research in race bunch cycling or, indeed, any sport which is characterised by the apparent non-linear aggregation (self organisation) of singular agents, forming an otherwise unattainable 'macro' end result.

\section{References}


Atkinson, G., Davison, R., Jeukendrup, A., and Passfield, L. (2003). Science and cycling: current knowledge and future directions for research. Journal of Sports Sciences, 9, (21), 767-787.

Chu, T., Wang, L., Chen, T., and Mu, S. (2006). Complex emergent dynamics of anisotropic swarms: Convergence vs oscillation. Chaos, Solitons and Fractals, 30, 875-885.

Edwards, A. G. (2007). Aerodynamic characteristics as determinants of the drafting effect in cycling. Medicine and Science in Sports and Exercise, 39, (1), 170-176.

Gazi, V. and Passino, K. M. (2004). Stability analysis of social foraging swarms. Systems, Man and Cybernetics, 34, 1539-1557.

Grehaigne, J. F., Richard, J. F., and Griffin, L. (2005). Teaching and Learning Team Sports and Games. New York: Routledge.

Haken, H. (1983). Synergetics: an introduction: non-equilibrium phase transitions and self-organisation in physics, chemistry and biology. Berlin: Springer-Verlag.

Haken, H., Kelso, J. A. S. and Bunz, H. (1985). A theoretical model of phase transitions in human hand movements. Biological Cybernetics, 51, (5). 347-356.

Hughes, M. D. (2004). Performance Analysis - a mathematical perspective. International Journal of Performance Analysis in Sport, 2, (4), 97-139.

Hughes, M. and Bartlett, R. M (2002). The use of performance indicators in performance analysis. Journal of Sport Sciences, 20, 739-754.

Hughes, M., Landridge, C., and Dawkins, N. (2001). Perturbation actions not leading to shots on goal in soccer. In M. Hughes (Edt.), Notational Analysis of Sport IV (pp. 23-32). Cardiff: UWIC.

Hughes, M., Dawkins, N., David, R., and Mills, J. (1998). The perturbation effect and goal opportunities in soccer. Journal of Sports Sciences, 16, 20.

James, N., Mellalieu, S., and Jones, N. (2005). The development of position-specific performance indicators in professional rugby union. Journal of Sports Sciences, 23, (1), 63-72. 
Lames, M. and McGarry, T. (2007). On the search for reliable performance indicators in game sports. International Journal of Performance Analysis in Sport, 7, (1), 62-79.

Lebed, F. (2006). Systems approach to games and competitive playing. European Journal of Sport Science, 6, 33-42.

McGarry, T (2009). Applied and theoretical perspectives of performance analysis in sport: Scientific issues and challenges. International Journal of Performance Analysis of Sport, 9, 128-140.

McGarry, T. (2006). Identifying patterns in squash contests using dynamical analysis and human perception. International Journal of Performance Analysis in Sport, 6, 134147.

Mcgarry, T. and Perl, J. (2004). Models of Sports Contests. In M. Hughes and I. M. Franks (Edt.), Notational Analysis of Sport (2 ${ }^{\text {nd }}$ Ed), (pp. 227-242). London: E and FN Spon.

McGarry, T., Khan, M.A. \& Franks, I.M. (1996). Analyzing championship squash matchplay: In search of a system description. In S. Haake (Edt.), The Engineering of Sport, (pp.263-269). Rotterdam: Balkema.

Mcgarry, T., Khan, M. A. and Franks, I. M. (1999). On the presence and absence of behavioural traits in sport: An example from championship squash match-play. Journal of Sports Sciences, 17, 297-311.

McGarry, T., Anderson, D., Hughes, M. and Franks, I. M. (2002). Sport competition as a dynamical self-organizing system. Journal of Sports Science. 20, 771-781.

O'Donoghue, P. (2007). Reliability issues in performance analysis. International Journal of Performance Analysis in Sport, 7, (1), 35-48.

Palut, Y. and Zanone, P. G. (2005). A dynamical analysis of tennis: Concepts and data. Journal of Sports Sciences, 10, (23), 1021-1032.

Parish, J. K. Viscido, S. V., and Grünbaum, D. (2002). Self-Organized fish schools: an examination of emergent properties. Biology Bulletin, 202, 296-305. 
Reed, D. and Hughes, M. (2006). An exploration of team sport as a dynamical system. International Journal of Performance Analysis in Sport, 2, (6), 114-125.

Worsfold, P. and MacBeth, K. (2009). Reliability of television broadcasting statistics in soccer. International Journal of Performance Analysis in Sport, 9, (3), 344-353. 